\title{
Sufficient Descent Conjugate Gradient Methods for Solving Convex Constrained Nonlinear Monotone Equations
}

\author{
San-Yang Liu, Yuan-Yuan Huang, and Hong-Wei Jiao \\ School of Mathematics and Statistics, Xidian University, Xi'an 710071, China \\ Correspondence should be addressed to San-Yang Liu; liusanyang@126.com and Yuan-Yuan Huang; yyuanhuang@126.com
}

Received 22 October 2013; Accepted 15 December 2013; Published 12 January 2014

Academic Editor: Zhongxiao Jia

Copyright (C) 2014 San-Yang Liu et al. This is an open access article distributed under the Creative Commons Attribution License, which permits unrestricted use, distribution, and reproduction in any medium, provided the original work is properly cited.

\begin{abstract}
Two unified frameworks of some sufficient descent conjugate gradient methods are considered. Combined with the hyperplane projection method of Solodov and Svaiter, they are extended to solve convex constrained nonlinear monotone equations. Their global convergence is proven under some mild conditions. Numerical results illustrate that these methods are efficient and can be applied to solve large-scale nonsmooth equations.
\end{abstract}

\section{Introduction}

Consider the constrained monotone equations

$$
F(x)=0, \quad x \in \Omega,
$$

where $F: R^{n} \rightarrow R^{n}$ is continuous and satisfies the following monotonicity:

$$
(F(x)-F(y))^{T}(x-y) \geq 0, \quad \forall x, y \in \Omega,
$$

and $\Omega \subset R^{n}$ is a nonempty closed convex set. Under these conditions, the solution set $X^{*}$ of problem (1) is convex [1]. This problem has many applications, such as the power flow equation $[2,3]$ and some variational inequality problems which can be converted into (1) by means of fixed point maps or normal maps if the underlying function satisfies some coercive conditions [4].

In recent years, the study of the iterative methods to solve problem (1) with $\Omega=R^{n}$ has received much attention. The pioneer work was introduced by Solodov and Svaiter in [5], where the proposed method was called inexact Newton method which combines elements of Newton method, proximal point method, and projection strategy and required that $F$ is differentiable. Its convergence was proven without any regularity assumptions. And a further study about its convergence properties was given by Zhou and Toh [6]. Then utilizing the projection strategy in [5], Zhou and Li extended the BFGS methods [7] and the limited memory BFGS methods [8] to solve problem (1) with $\Omega=R^{n}$. A significant improvement is that these methods converge globally without requiring the differentiability of $F$.

Conjugate gradient methods are another class of numerical methods [9-15] after spectral gradient methods [16-18] extended to solve problem (1), and the study of this aspect is just catching up. As is well known, conjugate gradient methods are very efficient to solve large-scale unconstrained nonlinear optimization problem

$$
\min f(x), \quad x \in R^{n},
$$

where $f$ is smooth, due to their simple iterations and their low memory requirements. In [19], they were divided into three categories, that is, early conjugate gradient methods, descent conjugate gradient methods, and sufficient descent conjugate gradient methods. Early conjugate gradient methods rarely ensure a (sufficient) descent condition

$$
g_{k}^{T} d_{k} \leq-c\left\|g_{k}\right\|^{2}, \quad \forall k \geq 0, c>0,
$$

where $g_{k}=g\left(x_{k}\right)$ is the gradient of $f$ at $x_{k}$ (the $k$ th iteration) and $d_{k}$ is a search direction, while the later two categories always satisfy the descent property. One wellknown sufficient descent conjugate gradient method, namely, CG_DESCENT, was presented by Hager and Zhang $[20,21]$ and satisfied the sufficient descent condition (4) with $c=7 / 8$. 
Inspired by Hager and Zhang's work, a unified framework of some sufficient descent conjugate gradient methods was presented in $[19,22]$. And by the use of Gram-Schmidt orthogonalization, the other unified framework of some sufficient descent conjugate gradient methods was presented in [23].

Although conjugate gradient methods have been investigated extensively for solving unconstrained optimization problems, the study of them to solve nonlinear monotone equations is relatively rare. For the unconstrained case of monotone equations, Cheng [10] first introduced a PRP type method which is a combination of the well-known PRP conjugate gradient method $[24,25]$ and the hyperplane projection method [5]. Then some derivative-free methods were presented [11-13] which also belong to the conjugate gradient scheme. More recently, Xiao and Zhu [9] presented a modified version of the CG_DESCENT method to solve the constrained nonlinear monotone equations. And under some mild conditions, they proved that their proposed method is globally convergent. We have mentioned that there are two unified frameworks of some sufficient descent conjugate gradient methods, and the CG_DESCENT method belongs to one unified framework. Since the CG_DESCENT method can be used to solve the constrained monotone equations, then, it is natural for us to think about the two unified frameworks. So, in this paper, we extend the conjugate gradient methods who belong to the two unified frameworks to solve the constrained monotone equations and do some numerical experiments to test their efficiency.

The rest of this paper is organized as follows. In Section 2, the motivation to investigate two unified frameworks of some sufficient descent conjugate gradient methods is given. Then these methods are developed to solve problem (1) and are described by a model algorithm. In Section 3, we prove the global convergence of the model algorithm under some mild conditions. In Section 4, we give several specific versions of the model algorithm, test them over some test problems, and compare their numerical performance with that of the conjugate gradient method proposed in [9]. Finally, some conclusions are given in Section 5.

\section{Motivation and Algorithms}

In this section, we simply describe the hyperplane projection method of Solodov and Svaiter and introduce two classes of sufficient descent conjugate gradient methods for solving large-scale unconstrained optimization problems. Combined with the hyperplane projection method, we extend sufficient descent conjugate gradient methods to solve large-scale constrained nonlinear equations (1).

For convenience, we first give the definition of projection operator $P_{\Omega}(\cdot)$ which is defined as a mapping from $R^{n}$ to its a nonempty closed convex subset $\Omega$ :

$$
P_{\Omega}(x)=\arg \min \{\|y-x\| \mid y \in \Omega\}, \quad \forall x \in R^{n} .
$$

And its two fundamental properties are

$$
\begin{gathered}
\left\|P_{\Omega}(x)-P_{\Omega}(y)\right\| \leq\|x-y\|, \quad \forall x, y \in R^{n}, \\
\left(x-P_{\Omega}(x)\right)^{T}\left(y-P_{\Omega}(x)\right) \leq 0, \quad \forall x \in R^{n}, y \in \Omega .
\end{gathered}
$$

Now, we recall the hyperplane projection method in [5] for the unconstrained case of problem (1). Let $x_{k}(k \geq 0)$ be the current iteration and $z_{k}=x_{k}+\alpha_{k} d_{k}$, where $\alpha_{k}$ is a step length obtained by means of a one-dimensional line search and $d_{k}$ is a search direction. If $x_{k}$ is not a solution and satisfies

$$
\left(x_{k}-z_{k}\right)^{T} F\left(z_{k}\right)>0,
$$

then the hyperplane

$$
H_{k}=\left\{x \in R^{n} \mid\left(x-z_{k}\right)^{T} F\left(z_{k}\right)=0\right\}
$$

strictly separates the current iteration $x_{k}$ from the solution set of problem (1). By the property (7) of the projection operator, it is not difficult to verify that

$$
P_{H_{k}}\left(x_{k}\right)=x_{k}-\frac{\left(x_{k}-z_{k}\right)^{T} F\left(z_{k}\right)}{\left\|F\left(z_{k}\right)\right\|^{2}} F\left(z_{k}\right)
$$

is closer to the solution set than the iteration $x_{k}$. Then the next iteration is generated by $x_{k+1}=P_{H_{k}}\left(x_{k}\right)$.

We consider the iterative scheme of conjugate gradient methods for solving the unconstrained optimization problem (3). For any given starting point $x_{0} \in R^{n}$, a sequence $\left\{x_{k}\right\}$ is generated by the following recursive relation:

$$
x_{k+1}=x_{k}+\alpha_{k} d_{k},
$$

where $\alpha_{k}$ is a steplength and $d_{k}$ is a descent direction. One way to generate $d_{k}$ is

$$
d_{k}= \begin{cases}-g_{k}, & \text { if } k=0, \\ -g_{k}+\beta_{k} d_{k-1}, & \text { if } k \geq 1,\end{cases}
$$

where $g_{k}=g\left(x_{k}\right)$ and $\beta_{k}$ is a scalar. The formula of $\beta_{k}$ in the CG_DESCENT method is defined as

$$
\beta_{k}^{H Z}=\frac{g_{k}^{T} y_{k-1}}{d_{k-1}^{T} y_{k-1}}-\frac{2\left\|y_{k-1}\right\|^{2}}{\left(d_{k-1}^{T} y_{k-1}\right)^{2}} g_{k}^{T} d_{k-1},
$$

where $y_{k-1}=g_{k}-g_{k-1}$. Then the direction $d_{k}$ from (12) satisfies the sufficient descent condition (4) with $c=7 / 8$. For more efficient versions of the CG_DESCENT method, please refer to $[26,27]$.

In $[19,22]$, a generalization of (13) was given by

$$
\beta_{k}^{G}=\frac{g_{k}^{T} b_{k}}{a_{k}}-\frac{C\left\|b_{k}\right\|^{2}}{a_{k}^{2}} g_{k}^{T} d_{k-1},
$$

where $a_{k} \in R, b_{k} \in R^{n}$, and $C>1 / 4$. Obviously, $\beta_{k}^{H Z}$ is a special case of (14) with $a_{k}=d_{k-1}^{T} y_{k-1}, b_{k}=y_{k-1}$, and $C=2$. More recently, Xiao and Zhu [9] presented a modified version of the CG_DESCENT method to solve 
the constrained problem (1). This work inspires us to extend the general case (14) to solve problem (1). So, we define

$$
d_{k}= \begin{cases}-F_{k}, & \text { if } k=0, \\ -F_{k}+\beta_{k} d_{k-1}, & \text { if } k \geq 1,\end{cases}
$$

where $F_{k}=F\left(x_{k}\right)$ and the scalar $\beta_{k}$ is defined as

$$
\beta_{k}=\frac{F_{k}^{T} b_{k}}{a_{k}}-\frac{\theta_{k}\left\|b_{k}\right\|^{2}}{a_{k}^{2}} F_{k}^{T} d_{k-1}
$$

with $\epsilon\left\|d_{k-1}\right\| \leq a_{k} \in R(\epsilon>0), \theta_{k}>1 / 2$, and $b_{k} \in R^{n}$. Moreover, the formula of $\beta_{k}$ proposed by Xiao and Zhu [9] corresponds to (16) with $\theta_{k}=2, a_{k}=d_{k-1}^{T} y_{k-1}^{*}$, and $b_{k}=y_{k-1}^{*}$, where $y_{k}^{*}=y_{k}+\lambda_{k} \alpha_{k}\left\|F_{k}\right\| d_{k}, y_{k}=F_{k+1}-F_{k}$, and $\lambda_{k}=1+$ $\left\|F_{k}\right\|^{-1} \max \left\{0,-\left(\alpha_{k} d_{k}^{T} y_{k}^{*} /\left\|\alpha_{k} d_{k}\right\|^{2}\right)\right\}$.

The other general way of producing sufficient descent conjugate gradient methods for solving the unconstrained optimization (3) was provided in [23]. By using the GramSchmidt orthogonalization, the search direction $d_{k}$ is generated by

$$
d_{k}= \begin{cases}-g_{k}, & \text { if } k=0, \\ -\left(1+\beta_{k} \frac{g_{k}^{T} d_{k-1}}{\left\|g_{k}\right\|^{2}}\right) g_{k}+\beta_{k} d_{k-1}, & \text { if } k \geq 1,\end{cases}
$$

where $\beta_{k}$ is a scalar, and its definition could be the same as that in (12). Obviously, it always satisfies $g_{k}^{T} d_{k}=-\left\|g_{k}\right\|^{2}$. In this paper, we will prove that the class of sufficient descent conjugate gradient methods can also be extended to solve problem (1) with the corresponding search direction $d_{k}$ defined as

$$
d_{k}= \begin{cases}-F_{k}, & \text { if } k=0, \\ -\left(1+\beta_{k} \frac{F_{k}^{T} d_{k-1}}{\left\|F_{k}\right\|^{2}}\right) F_{k}+\beta_{k} d_{k-1}, & \text { if } k \geq 1,\end{cases}
$$

where the formula of $\beta_{k}$ could be (16).

Now we introduce the two unified frameworks of some sufficient descent conjugate gradient methods to solve problem (1) by adopting the projection strategy in [5]. We state the steps of the model algorithm as follows.

\section{Algorithm 1.}

Step 0. Choose an initial point $x_{0} \in \Omega, \rho>0, \sigma \in(0,1)$, $t \in(0,1)$ and $\epsilon>0$. Set $k:=0$.

Step 1. If $\left\|F\left(x_{k}\right)\right\| \leq \epsilon$, stop. Otherwise, generate $d_{k}$ by certain iteration formula which satisfies sufficient descent condition.

Step 2. Let $\alpha_{k}$ be the largest $\alpha \in\left\{\rho t^{j} \mid j=0,1,2, \ldots\right\}$ such that

$$
-F\left(x_{k}+\alpha d_{k}\right)^{T} d_{k} \geq \sigma \alpha\left\|F\left(x_{k}+\alpha d_{k}\right)\right\|\left\|d_{k}\right\|^{2},
$$

and then compute $z_{k}=x_{k}+\alpha_{k} d_{k}$.

Step 3. Compute the new iterate $x_{k+1}$ by

$$
x_{k+1}=P_{\Omega}\left(x_{k}-\frac{\left(x_{k}-z_{k}\right)^{T} F\left(z_{k}\right)}{\left\|F\left(z_{k}\right)\right\|^{2}} F\left(z_{k}\right)\right) \text {. }
$$

Set $k:=k+1$. Go to Step 1 . (i) If the search direction $d_{k}$ in Step 1 is generated by the formula (15), we name the algorithm as Algorithm 1(a). And if the search direction $d_{k}$ in Step 1 is generated by the formula (18), we name the algorithm as Algorithm 1(b).

\section{Convergence Analysis}

In this section, we analyze the convergence properties of Algorithm 1. We first make the following assumptions.

Assumption 2. The mapping $F$ is $L$-Lipschitz continuous on the nonempty closed convex set $\Omega$; that is, there exists a constant $L>0$ such that

$$
\|F(x)-F(y)\| \leq L\|x-y\|, \quad \forall x, y \in \Omega .
$$

Assumption 3. The solution set $X^{*}$ of problem (1) is nonempty.

Assumption 4. The parameter $\beta_{k}$ satisfies inequality

$$
\left|\beta_{k}\right| \leq \frac{\gamma\left\|F_{k}\right\|}{\left\|d_{k-1}\right\|}
$$

where $\gamma$ is a positive number.

Assumption 4 is not difficult to satisfy. Taking the parameter $\beta_{k}$ in (15) as an example, if there exists a large number $M<\infty$ such that $\left\|b_{k}\right\| \leq M$ for all $k$, then it satisfies the inequality (22). In fact,

$$
\begin{aligned}
\left|\beta_{k}\right| & =\left|\frac{F_{k}^{T} b_{k}}{a_{k}}-\frac{\theta_{k}\left\|b_{k}\right\|^{2}}{a_{k}^{2}} F_{k}^{T} d_{k-1}\right| \\
& \leq\left|\frac{F_{k}^{T} b_{k}}{a_{k}}\right|+\left|\frac{\theta_{k}\left\|b_{k}\right\|^{2}}{a_{k}^{2}} F_{k}^{T} d_{k-1}\right| \\
& \leq \frac{\left\|F_{k}\right\|\left\|b_{k}\right\|}{\left|a_{k}\right|}+\frac{\theta_{k}\left\|b_{k}\right\|^{2}}{a_{k}^{2}}\left\|F_{k}\right\|\left\|d_{k-1}\right\| \\
& \leq \frac{\left\|F_{k}\right\| M}{\epsilon\left\|d_{k-1}\right\|}+\frac{\theta_{k} M^{2}}{\epsilon^{2}\left\|d_{k-1}\right\|^{2}}\left\|F_{k}\right\|\left\|d_{k-1}\right\| \\
& =\left(\frac{M}{\epsilon}+\frac{\theta_{k} M^{2}}{\epsilon^{2}}\right) \frac{\left\|F_{k}\right\|}{\left\|d_{k-1}\right\|} .
\end{aligned}
$$

The following two lemmas show that the search direction $d_{k}$, no matter from (15) or (18), satisfies the sufficient descent condition.

Lemma 5. If $a_{k} \neq 0, \theta_{k}>1 / 2$, and $d_{k}$ is generated by (15), then, for every $k \geq 0$,

$$
F_{k}^{T} d_{k} \leq-\left(1-\frac{1}{4 \theta_{k}}\right)\left\|F_{k}\right\|^{2}
$$


TABle 1: Numerical results for Problem 10.

\begin{tabular}{|c|c|c|c|c|c|}
\hline \multirow[t]{2}{*}{ Init } & \multirow[t]{2}{*}{ Method } & $\operatorname{Dim}=5000$ & $\operatorname{Dim}=10000$ & $\operatorname{Dim}=20000$ & $\begin{array}{l}\mathrm{Dim}=30000 \\
\text { Iter } / \mathrm{Nf} / \mathrm{CPU}\end{array}$ \\
\hline & & Iter/Nf/CPU & Iter/Nf/CPU & & \\
\hline \multirow{7}{*}{$x_{1}$} & CGD_XZ & $10 / 26 / 0.036$ & $10 / 26 / 0.052$ & $10 / 26 / 0.052$ & $10 / 26 / 0.071$ \\
\hline & Method 1 & $13 / 38 / 0.026$ & $13 / 38 / 0.038$ & $13 / 38 / 0.063$ & $13 / 38 / 0.089$ \\
\hline & Method 2 & $15 / 32 / 0.024$ & $19 / 38 / 0.047$ & $19 / 38 / 0.073$ & $31 / 74 / 0.179$ \\
\hline & Method 3 & $10 / 22 / 0.017$ & $10 / 22 / 0.023$ & $10 / 22 / 0.039$ & $10 / 22 / 0.052$ \\
\hline & Method 4 & $13 / 25 / 0.022$ & $13 / 25 / 0.028$ & $13 / 25 / 0.048$ & $13 / 25 / 0.061$ \\
\hline & Method 5 & $13 / 25 / 0.019$ & $13 / 25 / 0.028$ & $13 / 25 / 0.058$ & $13 / 25 / 0.063$ \\
\hline & Method 6 & $19 / 33 / 0.030$ & $19 / 34 / 0.040$ & $19 / 34 / 0.062$ & $29 / 67 / 0.162$ \\
\hline \multirow{7}{*}{$x_{2}$} & CGD_XZ & $5 / 10 / 0.007$ & $5 / 10 / 0.012$ & $5 / 10 / 0.020$ & $5 / 10 / 0.027$ \\
\hline & Method 1 & $7 / 19 / 0.011$ & $7 / 19 / 0.017$ & $7 / 19 / 0.029$ & 7/19/0.039 \\
\hline & Method 2 & $5 / 10 / 0.006$ & $5 / 10 / 0.010$ & $7 / 14 / 0.022$ & $7 / 14 / 0.035$ \\
\hline & Method 3 & $4 / 5 / 0.004$ & $4 / 5 / 0.006$ & 4/5/0.011 & $4 / 5 / 0.016$ \\
\hline & Method 4 & $5 / 6 / 0.006$ & $5 / 6 / 0.008$ & $5 / 6 / 0.014$ & $5 / 6 / 0.020$ \\
\hline & Method 5 & $5 / 6 / 0.005$ & $5 / 6 / 0.008$ & $5 / 6 / 0.013$ & $5 / 6 / 0.020$ \\
\hline & Method 6 & $5 / 6 / 0.005$ & $5 / 6 / 0.009$ & $6 / 8 / 0.017$ & $6 / 8 / 0.023$ \\
\hline \multirow{7}{*}{$x_{3}$} & CGD_XZ & $4 / 8 / 0.006$ & $4 / 8 / 0.010$ & $4 / 8 / 0.016$ & $4 / 8 / 0.021$ \\
\hline & Method 1 & $7 / 18 / 0.010$ & $7 / 18 / 0.015$ & $7 / 18 / 0.029$ & $7 / 18 / 0.037$ \\
\hline & Method 2 & $4 / 7 / 0.005$ & $4 / 7 / 0.008$ & $4 / 7 / 0.013$ & 4/7/0.018 \\
\hline & Method 3 & $3 / 4 / 0.003$ & $3 / 4 / 0.005$ & $3 / 4 / 0.009$ & $3 / 4 / 0.012$ \\
\hline & Method 4 & $4 / 5 / 0.005$ & $4 / 5 / 0.007$ & 4/5/0.011 & $4 / 5 / 0.015$ \\
\hline & Method 5 & $4 / 5 / 0.005$ & $4 / 5 / 0.006$ & $4 / 5 / 0.012$ & $4 / 5 / 0.015$ \\
\hline & Method 6 & $4 / 5 / 0.004$ & $4 / 5 / 0.007$ & $4 / 5 / 0.011$ & $4 / 5 / 0.014$ \\
\hline \multirow{7}{*}{$x_{4}$} & CGD_XZ & $15 / 47 / 0.027$ & $19 / 60 / 0.055$ & $17 / 51 / 0.080$ & $21 / 76 / 0.141$ \\
\hline & Method 1 & $14 / 47 / 0.025$ & $17 / 59 / 0.048$ & $17 / 58 / 0.077$ & $21 / 75 / 0.137$ \\
\hline & Method 2 & $14 / 28 / 0.016$ & $14 / 28 / 0.026$ & $14 / 28 / 0.047$ & $14 / 28 / 0.064$ \\
\hline & Method 3 & $18 / 28 / 0.022$ & $18 / 28 / 0.031$ & $18 / 28 / 0.053$ & $18 / 28 / 0.076$ \\
\hline & Method 4 & $14 / 21 / 0.017$ & $14 / 21 / 0.023$ & $14 / 21 / 0.043$ & $14 / 21 / 0.056$ \\
\hline & Method 5 & $10 / 15 / 0.012$ & $10 / 15 / 0.017$ & $10 / 15 / 0.028$ & $10 / 15 / 0.040$ \\
\hline & Method 6 & $21 / 33 / 0.025$ & $21 / 33 / 0.038$ & $21 / 33 / 0.060$ & $21 / 33 / 0.085$ \\
\hline \multirow{7}{*}{$x_{5}$} & CGD_XZ & $16 / 41 / 0.027$ & $24 / 65 / 0.066$ & 19/49/0.084 & 19/51/0.118 \\
\hline & Method 1 & $17 / 49 / 0.029$ & $17 / 55 / 0.044$ & $19 / 60 / 0.084$ & 18/58/0.111 \\
\hline & Method 2 & $13 / 26 / 0.017$ & $13 / 26 / 0.026$ & $13 / 26 / 0.045$ & $13 / 26 / 0.060$ \\
\hline & Method 3 & $23 / 35 / 0.028$ & $23 / 35 / 0.040$ & $23 / 35 / 0.067$ & $23 / 35 / 0.096$ \\
\hline & Method 4 & $22 / 34 / 0.025$ & $22 / 34 / 0.040$ & $22 / 34 / 0.068$ & $22 / 34 / 0.093$ \\
\hline & Method 5 & $21 / 30 / 0.023$ & $21 / 30 / 0.036$ & $21 / 30 / 0.060$ & $21 / 30 / 0.082$ \\
\hline & Method 6 & $25 / 41 / 0.033$ & $24 / 40 / 0.046$ & $24 / 40 / 0.070$ & $24 / 40 / 0.095$ \\
\hline \multirow{7}{*}{$x_{6}$} & CGD_XZ & $16 / 41 / 0.027$ & $23 / 56 / 0.060$ & $18 / 47 / 0.073$ & $19 / 51 / 0.112$ \\
\hline & Method 1 & $16 / 51 / 0.027$ & $15 / 47 / 0.038$ & $17 / 54 / 0.075$ & $17 / 54 / 0.101$ \\
\hline & Method 2 & $13 / 26 / 0.015$ & $13 / 26 / 0.026$ & $13 / 26 / 0.043$ & $13 / 26 / 0.058$ \\
\hline & Method 3 & $23 / 35 / 0.026$ & $23 / 35 / 0.040$ & $23 / 35 / 0.067$ & $23 / 35 / 0.092$ \\
\hline & Method 4 & $22 / 34 / 0.025$ & $22 / 34 / 0.042$ & $22 / 34 / 0.067$ & $22 / 34 / 0.088$ \\
\hline & Method 5 & $21 / 30 / 0.024$ & $21 / 30 / 0.035$ & $21 / 30 / 0.060$ & $21 / 30 / 0.085$ \\
\hline & Method 6 & $25 / 41 / 0.029$ & $24 / 40 / 0.042$ & $24 / 40 / 0.072$ & $24 / 40 / 0.097$ \\
\hline
\end{tabular}


Table 2: Numerical results for Problem 11.

\begin{tabular}{|c|c|c|c|c|c|}
\hline \multirow{2}{*}{ Init } & \multirow{2}{*}{ Method } & $\operatorname{Dim}=5000$ & $\operatorname{Dim}=10000$ & $\operatorname{Dim}=20000$ & $\operatorname{Dim}=30000$ \\
\hline & & Iter/Nf/CPU & Iter/Nf/CPU & Iter/Nf/CPU & Iter/Nf/CPU \\
\hline \multirow{7}{*}{$x_{1}$} & CGD_XZ & $5 / 12 / 0.019$ & $5 / 12 / 0.025$ & $5 / 12 / 0.033$ & $5 / 12 / 0.039$ \\
\hline & Method 1 & $7 / 21 / 0.023$ & $7 / 21 / 0.030$ & $7 / 21 / 0.039$ & $7 / 21 / 0.054$ \\
\hline & Method 2 & $5 / 13 / 0.015$ & $5 / 14 / 0.017$ & $5 / 14 / 0.028$ & $5 / 15 / 0.039$ \\
\hline & Method 3 & $12 / 25 / 0.022$ & $12 / 25 / 0.036$ & $12 / 25 / 0.047$ & $12 / 25 / 0.065$ \\
\hline & Method 4 & 5/8/0.009 & $5 / 8 / 0.012$ & $5 / 8 / 0.017$ & $5 / 8 / 0.025$ \\
\hline & Method 5 & $5 / 8 / 0.009$ & $5 / 8 / 0.014$ & $5 / 8 / 0.017$ & $5 / 8 / 0.025$ \\
\hline & Method 6 & $5 / 10 / 0.008$ & $5 / 11 / 0.022$ & $5 / 11 / 0.021$ & $5 / 12 / 0.030$ \\
\hline \multirow{7}{*}{$x_{2}$} & $\mathrm{CGD} \times \mathrm{XZ}$ & $3 / 6 / 0.004$ & $3 / 6 / 0.009$ & $3 / 6 / 0.013$ & $3 / 6 / 0.018$ \\
\hline & Method 1 & $6 / 17 / 0.011$ & $6 / 17 / 0.018$ & $6 / 17 / 0.027$ & $6 / 17 / 0.039$ \\
\hline & Method 2 & $3 / 6 / 0.004$ & $3 / 6 / 0.007$ & $3 / 6 / 0.012$ & $3 / 6 / 0.015$ \\
\hline & Method 3 & $9 / 18 / 0.014$ & $9 / 18 / 0.022$ & $9 / 18 / 0.034$ & $9 / 18 / 0.046$ \\
\hline & Method 4 & $3 / 4 / 0.004$ & $3 / 4 / 0.006$ & $3 / 4 / 0.009$ & $3 / 4 / 0.013$ \\
\hline & Method 5 & $3 / 4 / 0.004$ & $3 / 4 / 0.006$ & $3 / 4 / 0.010$ & $3 / 4 / 0.013$ \\
\hline & Method 6 & $3 / 4 / 0.004$ & $3 / 4 / 0.006$ & $3 / 4 / 0.009$ & $3 / 4 / 0.013$ \\
\hline \multirow{7}{*}{$x_{3}$} & CGD XZ & $14 / 41 / 0.028$ & $14 / 41 / 0.043$ & $14 / 41 / 0.073$ & $14 / 41 / 0.101$ \\
\hline & Method 1 & $5 / 13 / 0.008$ & $5 / 13 / 0.013$ & $5 / 13 / 0.021$ & $5 / 13 / 0.031$ \\
\hline & Method 2 & $13 / 37 / 0.022$ & $13 / 37 / 0.036$ & $13 / 37 / 0.061$ & $13 / 37 / 0.086$ \\
\hline & Method 3 & $10 / 20 / 0.015$ & $10 / 20 / 0.024$ & $10 / 20 / 0.040$ & $10 / 20 / 0.056$ \\
\hline & Method 4 & $14 / 28 / 0.021$ & $14 / 28 / 0.033$ & $14 / 28 / 0.054$ & $14 / 28 / 0.075$ \\
\hline & Method 5 & $14 / 28 / 0.021$ & $14 / 28 / 0.032$ & $14 / 28 / 0.055$ & $14 / 28 / 0.080$ \\
\hline & Method 6 & $14 / 28 / 0.020$ & $14 / 28 / 0.032$ & $14 / 28 / 0.052$ & $14 / 28 / 0.076$ \\
\hline \multirow{7}{*}{$x_{4}$} & CGD_XZ & $17 / 50 / 0.034$ & $17 / 50 / 0.051$ & $17 / 50 / 0.086$ & $17 / 50 / 0.124$ \\
\hline & Method 1 & $20 / 68 / 0.038$ & $17 / 50 / 0.044$ & $19 / 54 / 0.085$ & $18 / 51 / 0.118$ \\
\hline & Method 2 & $18 / 44 / 0.025$ & $16 / 38 / 0.040$ & $17 / 42 / 0.072$ & $17 / 42 / 0.096$ \\
\hline & Method 3 & $20 / 40 / 0.028$ & $21 / 42 / 0.043$ & $20 / 40 / 0.075$ & $18 / 36 / 0.094$ \\
\hline & Method 4 & $15 / 30 / 0.020$ & $15 / 30 / 0.034$ & $15 / 30 / 0.057$ & $15 / 30 / 0.080$ \\
\hline & Method 5 & $16 / 32 / 0.026$ & $16 / 32 / 0.039$ & $16 / 32 / 0.063$ & $16 / 32 / 0.083$ \\
\hline & Method 6 & $22 / 41 / 0.031$ & $19 / 38 / 0.042$ & $19 / 38 / 0.066$ & $19 / 38 / 0.097$ \\
\hline \multirow{7}{*}{$x_{5}$} & CGD_XZ & $21 / 59 / 0.044$ & $21 / 68 / 0.068$ & $17 / 52 / 0.089$ & $16 / 42 / 0.106$ \\
\hline & Method 1 & $19 / 61 / 0.033$ & $18 / 56 / 0.051$ & $17 / 50 / 0.078$ & $16 / 48 / 0.107$ \\
\hline & Method 2 & $12 / 23 / 0.016$ & $12 / 23 / 0.023$ & $12 / 23 / 0.043$ & $12 / 23 / 0.063$ \\
\hline & Method 3 & $17 / 34 / 0.024$ & $17 / 34 / 0.036$ & $17 / 34 / 0.063$ & $17 / 34 / 0.088$ \\
\hline & Method 4 & $32 / 52 / 0.040$ & $32 / 52 / 0.070$ & $31 / 50 / 0.101$ & $31 / 50 / 0.148$ \\
\hline & Method 5 & $21 / 35 / 0.028$ & $21 / 35 / 0.043$ & $21 / 35 / 0.073$ & $21 / 35 / 0.101$ \\
\hline & Method 6 & $29 / 39 / 0.034$ & $27 / 36 / 0.048$ & $27 / 36 / 0.079$ & $27 / 36 / 0.112$ \\
\hline \multirow{7}{*}{$x_{6}$} & CGD_XZ & $21 / 59 / 0.041$ & $22 / 68 / 0.071$ & $20 / 62 / 0.097$ & $16 / 42 / 0.104$ \\
\hline & Method 1 & $17 / 59 / 0.035$ & $19 / 67 / 0.058$ & $18 / 63 / 0.092$ & $18 / 52 / 0.118$ \\
\hline & Method 2 & $14 / 27 / 0.020$ & $14 / 27 / 0.031$ & $14 / 27 / 0.050$ & $14 / 27 / 0.074$ \\
\hline & Method 3 & $17 / 34 / 0.023$ & $17 / 34 / 0.040$ & $17 / 34 / 0.060$ & $17 / 34 / 0.090$ \\
\hline & Method 4 & $30 / 48 / 0.042$ & $31 / 50 / 0.060$ & $31 / 50 / 0.103$ & $31 / 50 / 0.143$ \\
\hline & Method 5 & $21 / 35 / 0.028$ & $21 / 35 / 0.040$ & $21 / 35 / 0.073$ & $21 / 35 / 0.101$ \\
\hline & Method 6 & $29 / 39 / 0.032$ & $27 / 36 / 0.046$ & $27 / 36 / 0.083$ & $27 / 36 / 0.112$ \\
\hline
\end{tabular}

Proof. Since $d_{0}=-F_{0}$, then $F_{0}^{T} d_{0}=-\left\|F_{0}\right\|^{2}$ which satisfies (24). For every $k \geq 1$, multiplying (15) by $F_{k}$, we have

$$
\begin{aligned}
& F_{k}^{T} d_{k} \\
& \quad=-\left\|F_{k}\right\|^{2}+\beta_{k} F_{k}^{T} d_{k-1}
\end{aligned}
$$

$$
\begin{aligned}
& =-\left\|F_{k}\right\|^{2}+\frac{F_{k}^{T} b_{k}}{a_{k}} F_{k}^{T} d_{k-1}-\frac{\theta_{k}\left\|b_{k}\right\|^{2}}{a_{k}^{2}}\left(F_{k}^{T} d_{k-1}\right)^{2} \\
& =\frac{-\left\|F_{k}\right\|^{2} a_{k}^{2}+a_{k}\left(F_{k}^{T} b_{k}\right)\left(F_{k}^{T} d_{k-1}\right)-\theta_{k}\left\|b_{k}\right\|^{2}\left(F_{k}^{T} d_{k-1}\right)^{2}}{a_{k}^{2}} .
\end{aligned}
$$


TABle 3: Numerical results for Problem 12.

\begin{tabular}{|c|c|c|c|c|c|}
\hline \multirow{2}{*}{ Init } & \multirow{2}{*}{ Method } & $\operatorname{Dim}=5000$ & $\operatorname{Dim}=10000$ & $\operatorname{Dim}=20000$ & $\operatorname{Dim}=30000$ \\
\hline & & Iter/Nf/CPU & Iter/Nf/CPU & Iter/Nf/CPU & Iter/Nf/CPU \\
\hline \multirow{7}{*}{$x_{1}$} & CGD_XZ & $24 / 66 / 0.095$ & $24 / 66 / 0.130$ & $21 / 60 / 0.205$ & $21 / 60 / 0.308$ \\
\hline & Method 1 & $14 / 34 / 0.037$ & $15 / 38 / 0.070$ & $15 / 46 / 0.146$ & $17 / 56 / 0.250$ \\
\hline & Method 2 & $26 / 66 / 0.063$ & 28/75/0.126 & $32 / 102 / 0.317$ & $41 / 136 / 0.612$ \\
\hline & Method 3 & $30 / 49 / 0.060$ & $30 / 49 / 0.099$ & $30 / 49 / 0.191$ & $30 / 49 / 0.278$ \\
\hline & Method 4 & $23 / 43 / 0.050$ & $23 / 43 / 0.085$ & $22 / 42 / 0.159$ & $22 / 42 / 0.224$ \\
\hline & Method 5 & $24 / 44 / 0.051$ & $23 / 43 / 0.083$ & $22 / 42 / 0.160$ & $22 / 42 / 0.222$ \\
\hline & Method 6 & $25 / 61 / 0.060$ & 28/76/0.127 & $33 / 103 / 0.320$ & $38 / 120 / 0.538$ \\
\hline \multirow{7}{*}{$x_{2}$} & CGD_XZ & $19 / 55 / 0.056$ & 19/55/0.112 & $18 / 53 / 0.177$ & $18 / 53 / 0.260$ \\
\hline & Method 1 & $16 / 40 / 0.041$ & $18 / 54 / 0.090$ & $13 / 33 / 0.112$ & $14 / 41 / 0.189$ \\
\hline & Method 2 & $18 / 36 / 0.038$ & $18 / 36 / 0.068$ & $18 / 36 / 0.127$ & $18 / 36 / 0.186$ \\
\hline & Method 3 & $19 / 37 / 0.040$ & $19 / 37 / 0.070$ & $18 / 36 / 0.130$ & $18 / 36 / 0.188$ \\
\hline & Method 4 & $19 / 37 / 0.040$ & $19 / 37 / 0.073$ & $18 / 36 / 0.128$ & $18 / 36 / 0.188$ \\
\hline & Method 5 & $19 / 37 / 0.042$ & $19 / 37 / 0.072$ & $18 / 36 / 0.127$ & $18 / 36 / 0.193$ \\
\hline & Method 6 & $18 / 36 / 0.039$ & $18 / 36 / 0.069$ & $18 / 36 / 0.128$ & $18 / 36 / 0.188$ \\
\hline \multirow{7}{*}{$x_{3}$} & CGD_XZ & $20 / 57 / 0.059$ & $19 / 54 / 0.095$ & $19 / 54 / 0.183$ & $19 / 54 / 0.266$ \\
\hline & Method 1 & $15 / 43 / 0.041$ & $12 / 28 / 0.050$ & $13 / 34 / 0.111$ & $18 / 55 / 0.251$ \\
\hline & Method 2 & $19 / 37 / 0.040$ & $19 / 37 / 0.068$ & $20 / 39 / 0.141$ & $20 / 39 / 0.202$ \\
\hline & Method 3 & $19 / 36 / 0.039$ & $19 / 36 / 0.068$ & $19 / 36 / 0.130$ & 19/36/0.195 \\
\hline & Method 4 & $21 / 40 / 0.046$ & $21 / 40 / 0.081$ & $21 / 40 / 0.147$ & $21 / 40 / 0.213$ \\
\hline & Method 5 & $21 / 40 / 0.046$ & $21 / 40 / 0.081$ & $21 / 40 / 0.148$ & $21 / 40 / 0.214$ \\
\hline & Method 6 & $19 / 37 / 0.041$ & $19 / 37 / 0.073$ & $18 / 36 / 0.128$ & $18 / 36 / 0.186$ \\
\hline \multirow{7}{*}{$x_{4}$} & CGD_XZ & $19 / 54 / 0.054$ & $19 / 54 / 0.098$ & $19 / 54 / 0.181$ & $19 / 54 / 0.263$ \\
\hline & Method 1 & $12 / 28 / 0.029$ & $12 / 29 / 0.054$ & $16 / 51 / 0.163$ & $14 / 36 / 0.175$ \\
\hline & Method 2 & $19 / 37 / 0.041$ & $19 / 37 / 0.072$ & 20/39/0.142 & $20 / 39 / 0.205$ \\
\hline & Method 3 & $19 / 36 / 0.041$ & 19/36/0.077 & $19 / 36 / 0.134$ & 19/36/0.190 \\
\hline & Method 4 & $20 / 38 / 0.043$ & $20 / 38 / 0.076$ & $20 / 38 / 0.141$ & $20 / 38 / 0.207$ \\
\hline & Method 5 & $20 / 38 / 0.043$ & $20 / 38 / 0.075$ & $20 / 38 / 0.141$ & $20 / 38 / 0.204$ \\
\hline & Method 6 & $19 / 37 / 0.040$ & $19 / 37 / 0.068$ & $18 / 36 / 0.128$ & $18 / 36 / 0.188$ \\
\hline \multirow{7}{*}{$x_{5}$} & CGD_XZ & $20 / 57 / 0.058$ & $20 / 57 / 0.101$ & $20 / 57 / 0.190$ & $20 / 57 / 0.273$ \\
\hline & Method 1 & $17 / 40 / 0.040$ & $16 / 49 / 0.080$ & $12 / 28 / 0.097$ & $12 / 28 / 0.141$ \\
\hline & Method 2 & $19 / 37 / 0.040$ & $19 / 37 / 0.072$ & 19/37/0.138 & 20/39/0.211 \\
\hline & Method 3 & $20 / 38 / 0.042$ & $20 / 38 / 0.074$ & $20 / 38 / 0.143$ & $20 / 38 / 0.204$ \\
\hline & Method 4 & $21 / 40 / 0.046$ & $21 / 40 / 0.075$ & $21 / 40 / 0.149$ & $21 / 40 / 0.212$ \\
\hline & Method 5 & $21 / 40 / 0.049$ & $21 / 40 / 0.077$ & $21 / 40 / 0.149$ & $21 / 40 / 0.212$ \\
\hline & Method 6 & $20 / 39 / 0.043$ & $20 / 39 / 0.076$ & 20/39/0.142 & 19/38/0.196 \\
\hline \multirow{7}{*}{$x_{6}$} & CGD_XZ & $19 / 54 / 0.052$ & $19 / 54 / 0.094$ & 19/54/0.182 & $19 / 54 / 0.264$ \\
\hline & Method 1 & $15 / 40 / 0.038$ & $12 / 28 / 0.052$ & $12 / 30 / 0.097$ & $17 / 51 / 0.233$ \\
\hline & Method 2 & $19 / 37 / 0.038$ & $19 / 37 / 0.068$ & 19/37/0.136 & $20 / 39 / 0.203$ \\
\hline & Method 3 & $19 / 36 / 0.040$ & $19 / 36 / 0.075$ & $19 / 36 / 0.133$ & 19/36/0.197 \\
\hline & Method 4 & $20 / 38 / 0.043$ & $20 / 38 / 0.076$ & 20/38/0.140 & $20 / 38 / 0.202$ \\
\hline & Method 5 & $20 / 38 / 0.042$ & $20 / 38 / 0.075$ & $20 / 38 / 0.142$ & $20 / 38 / 0.206$ \\
\hline & Method 6 & $19 / 37 / 0.040$ & $19 / 37 / 0.072$ & $19 / 37 / 0.133$ & $18 / 36 / 0.185$ \\
\hline
\end{tabular}


TABLE 4: Numerical results for Problem 13.

\begin{tabular}{lccr}
\hline Method & $\begin{array}{c}\text { Init }=x_{1} \\
\text { Iter/Nf/CPU }\end{array}$ & $\begin{array}{c}\text { Init }=x_{2} \\
\text { Iter/Nf/CPU }\end{array}$ & $\begin{array}{c}\text { Init }=x_{3} \\
\text { Iter/Nf/CPU }\end{array}$ \\
\hline CGD_XZ & $8032 / 100720 / 3.926$ & $6960 / 88626 / 3.395$ & $6608 / 83404 / 3.206$ \\
Method 1 & $14474 / 81644 / 4.073$ & $14776 / 83135 / 4.152$ & $14105 / 79821 / 3.985$ \\
Method 2 & $14547 / 80367 / 3.784$ & $14535 / 79834 / 3.782$ & $14233 / 78523 / 3.724$ \\
Method 3 & $15974 / 67287 / 3.949$ & $15950 / 67116 / 3.929$ & $15522 / 64072 / 3.830$ \\
Method 4 & $14289 / 77460 / 4.229$ & $14583 / 78517 / 4.262$ & $14034 / 76578 / 4.094$ \\
Method 5 & $14633 / 79306 / 4.089$ & $14585 / 78940 / 4.017$ & $12500 / 60647 / 3.319$ \\
Method 6 & $12394 / 61365 / 3.314$ & $13192 / 64579 / 3.495$ & Init $=x_{6}$ \\
\hline Method & Init $=x_{4}$ & Init $=x_{5}$ & 1 Iter/Nf/CPU \\
\hline CGD_XZ & Iter/Nf/CPU & Iter/Nf/CPU & $7583 / 92362 / 3.612$ \\
Method 1 & $8018 / 100772 / 3.874$ & $118 / 1232 / 0.050$ & $14523 / 81523 / 4.078$ \\
Method 2 & $14482 / 81707 / 4.059$ & $125 / 445 / 0.029$ & $14829 / 81427 / 3.871$ \\
Method 3 & $14490 / 80119 / 3.795$ & $65 / 209 / 0.014$ & $15802 / 66762 / 3.889$ \\
Method 4 & $15571 / 65689 / 3.826$ & $125 / 586 / 0.032$ & $14716 / 79737 / 4.267$ \\
Method 5 & $14618 / 78866 / 4.250$ & $132 / 450 / 0.032$ & $14530 / 79232 / 4.032$ \\
Method 6 & $14605 / 79474 / 4.059$ & $127 / 440 / 0.030$ & $12651 / 63054 / 3.378$ \\
\hline
\end{tabular}

TABLE 5: Number of times when each method was the fastest.

\begin{tabular}{lccc}
\hline Method & Iteration metric & $\begin{array}{c}\text { Function } \\
\text { evaluation metric }\end{array}$ & Time metric \\
\hline CGD_XZ & 17 & 0 & 3 \\
Method 1 & 28 & 16 & 19 \\
Method 2 & 26 & 22 & 24 \\
Method 3 & 12 & 19 & 13 \\
Method 4 & 12 & 15 & 11 \\
Method 5 & 12 & 15 & 10 \\
Method 6 & 10 & 16 & 11 \\
\hline
\end{tabular}

Denote $u_{k}=a_{k} F_{k} / \sqrt{2 \theta_{k}}$ and $v_{k}=\sqrt{2 \theta_{k}}\left(F_{k}^{T} d_{k-1}\right) b_{k}$. By applying the inequality $u_{k}^{T} v_{k} \leq 1 / 2\left(\left\|u_{k}\right\|^{2}+\left\|v_{k}\right\|^{2}\right)$ to the second term in (25), we obtain (24).

The lemma above is similar to Theorem 1.1 in [20]. And from this lemma, we can see that the descent property of $d_{k}$ from (15) is independent of any line search and choices of the parameters $a_{k}$ and $b_{k}$. While different choices of the parameters $a_{k}, b_{k}$, and $\theta_{k}$ may yield very different numerical behaviors.

Lemma 6. Let $\left\{d_{k}\right\}$ be the sequence generated by (18), and then, for all $k \geq 0$, it holds that

$$
F_{k}^{T} d_{k}=-\left\|F_{k}\right\|^{2} \leq-\left(1-\frac{1}{4 \theta_{k}}\right)\left\|F_{k}\right\|^{2}
$$

where $\theta_{k}>1 / 2$.
Proof. The desired result is very easy to obtain. In fact, if $k=$ 0 , it is clear that $F_{0}^{T} d_{0}=-\left\|F_{0}\right\|^{2} \leq-\left(1-\left(1 / 4 \theta_{0}\right)\right)\left\|F_{0}\right\|^{2}$. If $k \geq 1$, we have

$$
\begin{aligned}
F_{k}^{T} d_{k} & =F_{k}^{T}\left(-\left(1+\beta_{k} \frac{F_{k}^{T} d_{k-1}}{\left\|F_{k}\right\|^{2}}\right) F_{k}+\beta_{k} d_{k-1}\right) \\
& =-\left\|F_{k}\right\|^{2} \leq-\left(1-\frac{1}{4 \theta_{k}}\right)\left\|F_{k}\right\|^{2} .
\end{aligned}
$$

The lemma above indicates that the descent property of $d_{k}$ from (18) is independent of the choices of $\beta_{k}$.

Lemma 7. Suppose Assumptions 2 and 3 hold. Let $\alpha_{k}$ be the steplength involved in Algorithm 1 , and let sequences $\left\{x_{k}\right\}$ and $\left\{z_{k}\right\}$ be generated by Algorithm 1. Then steplength $\alpha_{k}$ is well defined and satisfies the following inequality:

$$
\alpha_{k} \geq \min \left\{\rho, \frac{t\left(1-\left(1 / 4 \theta_{k}\right)\right)}{L+\sigma\left\|F\left(x_{k}+\alpha_{k} t^{-1} d_{k}\right)\right\|} \frac{\left\|F_{k}\right\|^{2}}{\left\|d_{k}\right\|^{2}}\right\} .
$$

Proof. Suppose that, at $k$ th iteration, $x_{k}$ is not a solution, that is, $F_{k} \neq 0$, and, for all $j=0,1, \ldots$, inequality (19) fails to hold, and then

$$
-F\left(x_{k}+\rho t^{j} d_{k}\right)^{T} d_{k}<\sigma \rho t^{j}\left\|F\left(x_{k}+\rho t^{j} d_{k}\right)\right\|\left\|d_{k}\right\|^{2} .
$$

Since $F$ is continuous, taking the limits with respect to $j$ on the both sides of (29) yields

$$
-F\left(x_{k}\right)^{T} d_{k} \leq 0,
$$

which contradicts Lemmas 5 and 6 . So, the step length $\alpha_{k}$ is well defined and can be determined within a finite number of trials. 
Now, we prove inequality (28). If $\alpha_{k} \neq \rho$, then by using the selection of $\alpha_{k}$, we have

$$
-F\left(x_{k}+\alpha_{k} t^{-1} d_{k}\right)^{T} d_{k}<\sigma \alpha_{k} t^{-1}\left\|F\left(x_{k}+\alpha_{k} t^{-1} d_{k}\right)\right\|\left\|d_{k}\right\|^{2} .
$$

Combining it with (24), (26), and the Lipschitz continuity of $F$ yields

$$
\begin{aligned}
(1- & \left.\frac{1}{4 \theta_{k}}\right)\left\|F_{k}\right\|^{2} \\
& \leq-F_{k}^{T} d_{k} \\
& =\left(F\left(x_{k}+\alpha_{k} t^{-1} d_{k}\right)-F_{k}\right)^{T} d_{k}-F\left(x_{k}+\alpha_{k} t^{-1} d_{k}\right)^{T} d_{k} \\
& \leq L \alpha_{k} t^{-1}\left\|d_{k}\right\|^{2}+\sigma \alpha_{k} t^{-1}\left\|F\left(x_{k}+\alpha_{k} t^{-1} d_{k}\right)\right\|\left\|d_{k}\right\|^{2} .
\end{aligned}
$$

From Lemmas 5 and 6, we have that

$$
\begin{aligned}
\left\|d_{k}\right\|^{2} & =\left\|d_{k}+F_{k}-F_{k}\right\|^{2} \\
& =\left\|d_{k}+F_{k}\right\|^{2}-2 F_{k}^{T} d_{k}-\left\|F_{k}\right\|^{2} \\
& \geq\left(1-\frac{1}{2 \theta_{k}}\right)\left\|F_{k}\right\|^{2} .
\end{aligned}
$$

Since $F_{k} \neq 0$, then (33) indicates $d_{k} \neq 0$. So, it follows from inequality (32) that

$$
\alpha_{k} \geq \frac{t\left(1-\left(1 / 4 \theta_{k}\right)\right)}{L+\sigma\left\|F\left(x_{k}+\alpha_{k} t^{-1} d_{k}\right)\right\|} \frac{\left\|F_{k}\right\|^{2}}{\left\|d_{k}\right\|^{2}} .
$$

Then inequality (28) is obtained.

Lemma 8. Let $x^{*} \in X^{*}$ and let sequences $\left\{x_{k}\right\}$ and $\left\{F_{k}\right\}$ be generated by Algorithm 1 . Then one has

$$
\left\|x_{k+1}-x^{*}\right\|^{2} \leq\left\|x_{k}-x^{*}\right\|^{2}-\sigma^{2}\left\|x_{k}-z_{k}\right\|^{4} .
$$

Furthermore,

$$
\lim _{k \rightarrow \infty}\left\|x_{k}-z_{k}\right\|=\lim _{k \rightarrow \infty} \alpha_{k}\left\|d_{k}\right\|=0
$$

And there exists a positive number $M$ such that $\left\|F_{k}\right\| \leq M$ and $\left\|F\left(x_{k}+\alpha_{k} t^{-1} d_{k}\right)\right\| \leq M$ for all $k \geq 0$.

Proof. Since $x^{*} \in X^{*}$, then $F\left(x^{*}\right)=0$ and $P_{\Omega}\left(x^{*}\right)=x^{*}$. Since the mapping $F$ is monotone, then $F\left(z_{k}\right)^{T}\left(z_{k}-x^{*}\right) \geq 0$; further,

$$
F\left(z_{k}\right)^{T}\left(x_{k}-x^{*}\right) \geq F\left(z_{k}\right)^{T}\left(x_{k}-z_{k}\right) .
$$

By using (19) and $z_{k}=x_{k}+\alpha_{k} d_{k}$, we have

$$
F\left(z_{k}\right)^{T}\left(x_{k}-z_{k}\right)=-\alpha_{k} F\left(z_{k}\right)^{T} d_{k} \geq \sigma \alpha_{k}^{2}\left\|d_{k}\right\|^{2}\left\|F\left(z_{k}\right)\right\|,
$$

which implies that

$$
\frac{F\left(z_{k}\right)^{T}\left(x_{k}-z_{k}\right)}{\left\|F\left(z_{k}\right)\right\|} \geq \sigma \alpha_{k}^{2}\left\|d_{k}\right\|^{2}=\sigma\left\|x_{k}-z_{k}\right\|^{2} .
$$

Obviously, $F\left(z_{k}\right)^{T}\left(x_{k}-z_{k}\right) \geq 0$. By using the property (7) of the projection operator $P_{\Omega}(\cdot)$ and (37), we have

$$
\begin{aligned}
\| x_{k+1}- & x^{*} \|^{2} \\
& =\left\|P_{\Omega}\left(x_{k}-\frac{F\left(z_{k}\right)^{T}\left(x_{k}-z_{k}\right)}{\left\|F\left(z_{k}\right)\right\|^{2}} F\left(z_{k}\right)\right)-P_{\Omega}\left(x^{*}\right)\right\|^{2} \\
& \leq\left\|x_{k}-\frac{F\left(z_{k}\right)^{T}\left(x_{k}-z_{k}\right)}{\left\|F\left(z_{k}\right)\right\|^{2}} F\left(z_{k}\right)-x^{*}\right\|^{2} \\
& \leq\left\|x_{k}-x^{*}\right\|^{2}-\frac{\left(F\left(z_{k}\right)^{T}\left(x_{k}-z_{k}\right)\right)^{2}}{\left\|F\left(z_{k}\right)\right\|^{2}}
\end{aligned}
$$

Substituting the second term in (40) by (39), inequality (35) follows.

The inequality (35) shows that the sequence $\left\{\left\|x_{k}-x^{*}\right\|\right\}$ is convergent, and then taking the limits with respect to $k$ on the both sides of (35) yields (36).

Since $F$ is Lipschitz continuous, then from (35), we have

$$
\left\|F_{k}\right\|=\left\|F\left(x_{k}\right)-F\left(x^{*}\right)\right\| \leq L\left\|x_{k}-x^{*}\right\| \leq L\left\|x_{0}-x^{*}\right\| .
$$

And from (36), we know that there exists a positive number $M^{\prime}$ such that $\alpha_{k}\left\|d_{k}\right\| \leq M^{\prime}$, and then

$$
\begin{aligned}
\left\|F\left(x_{k}+\alpha_{k} t^{-1} d_{k}\right)\right\| & =\left\|F\left(x_{k}+\alpha_{k} t^{-1} d_{k}\right)-F\left(x^{*}\right)\right\| \\
& \leq L\left\|x_{k}+\alpha_{k} t^{-1} d_{k}-x^{*}\right\| \\
& \leq L\left\|x_{0}-x^{*}\right\|+L M^{\prime} t^{-1} .
\end{aligned}
$$

Denote $M=L\left\|x_{0}-x^{*}\right\|+L M^{\prime} t^{-1}$; we have that $\left\|F_{k}\right\| \leq M$ and $\left\|F\left(x_{k}+\alpha_{k} t^{-1} d_{k}\right)\right\| \leq M$.

Theorem 9. Let Assumption 4 hold and let $\left\{d_{k}\right\}$ be a sequence generated by Algorithm 1. Then

$$
\left\|d_{k}\right\| \leq(1+2 \gamma)\left\|F_{k}\right\|
$$

And one has

$$
\liminf _{k \rightarrow \infty}\left\|F_{k}\right\|=0
$$

Proof. If $d_{k}$ is generated by (15), we have

$$
\left\|d_{k}\right\| \leq\left\|F_{k}\right\|+\left|\beta_{k}\right|\left\|d_{k-1}\right\| \leq(1+\gamma)\left\|F_{k}\right\|,
$$

which satisfies (43). If $d_{k}$ is generated by (18), then

$$
\left\|d_{k}\right\| \leq\left\|F_{k}\right\|+2\left|\beta_{k}\right|\left\|d_{k-1}\right\| \text {. }
$$


The inequality (43) is obtained easily. From Lemma 8, we know that there exists $0<M<\infty$ such that $\left\|F_{k}\right\| \leq M$, and then

$$
\left\|d_{k}\right\| \leq(1+2 \gamma) M
$$
that

Suppose (44) does not hold, then there exists $\epsilon>0$ such

$$
\left\|F_{k}\right\| \geq \epsilon, \quad \forall k \geq 0
$$

From (33), we have that $\left\|d_{k}\right\| \geq \sqrt{\left(1-1 /\left(2 \theta_{k}\right)\right)}\left\|F_{k}\right\|$, which implies

$$
\left\|d_{k}\right\| \geq \sqrt{\left(1-\frac{1}{\left(2 \theta_{k}\right)}\right)} \epsilon, \quad \forall k \geq 0 .
$$

By inequalities (28), (47), (48), and (49), we have

$$
\begin{aligned}
\alpha_{k}\left\|d_{k}\right\| \geq & \min \left\{\rho, \frac{t\left(1-\left(1 / 4 \theta_{k}\right)\right)}{L+\sigma\left\|F\left(x_{k}+\alpha_{k} t^{-1} d_{k}\right)\right\|} \frac{\left\|F_{k}\right\|^{2}}{\left\|d_{k}\right\|^{2}}\right\}\left\|d_{k}\right\| \\
\geq & \min \left\{\rho, \frac{t\left(1-\left(1 / 4 \theta_{k}\right)\right)}{L+\sigma M} \frac{\epsilon^{2}}{(1+2 \gamma)^{2} M^{2}}\right\} \\
& \times \sqrt{\left(1-\frac{1}{\left(2 \theta_{k}\right)}\right)} \epsilon \\
> & 0 .
\end{aligned}
$$

This contradicts (36). So the conclusion (44) holds.

\section{Numerical Experiments}

In this section, we give some specific versions of Algorithm 1 and investigate their numerical behaviors. Let us review the HS conjugate gradient method [28]. It generates search direction $d_{k}$ by (12) and parameter $\beta_{k}$ by

$$
\beta_{k}^{H S}=\frac{g_{k}^{T} y_{k-1}}{d_{k-1}^{T} y_{k-1}} .
$$

Among early conjugate gradient methods, the HS method is a relatively efficient one. And many conjugate gradient methods are its improved versions, such as the well-known CG_DESCENT method. Now based on the HS method and Assumption 4, we give several specific versions of Algorithm 1(a) as follows.

Method 1. Consider Algorithm 1(a) with

$$
\begin{aligned}
\beta_{k}^{1}= & \frac{F_{k}^{T} y_{k-1}}{\max \left\{0.5 d_{k-1}^{T} y_{k-1}+0.5\left\|F_{k-1}\right\|^{2}, \epsilon\left\|d_{k-1}\right\|\right\}} \\
& -\frac{2\left\|y_{k-1}\right\|^{2}}{\left(\max \left\{0.5 d_{k-1}^{T} y_{k-1}+0.5\left\|F_{k-1}\right\|^{2}, \epsilon\left\|d_{k-1}\right\|\right\}\right)^{2}} \\
& \times F_{k}^{T} d_{k-1} .
\end{aligned}
$$

Method 2. Consider Algorithm 1(a) with

$$
\begin{aligned}
\beta_{k}^{2}= & \frac{F_{k}^{T} y_{k-1}}{\max \left\{\max \left\{d_{k-1}^{T} y_{k-1},\left\|F_{k-1}\right\|^{2}\right\}, \epsilon\left\|d_{k-1}\right\|\right\}} \\
& -\frac{2\left\|y_{k-1}\right\|^{2}}{\left(\max \left\{\max \left\{d_{k-1}^{T} y_{k-1},\left\|F_{k-1}\right\|^{2}\right\}, \epsilon\left\|d_{k-1}\right\|\right\}\right)^{2}} \\
& \times F_{k}^{T} d_{k-1} .
\end{aligned}
$$

Method 3. Consider Algorithm 1(a) with

$$
\begin{aligned}
\beta_{k}^{3}= & \frac{F_{k}^{T} y_{k-1}^{*}}{\max \left\{d_{k-1}^{T} y_{k-1}^{*}, \epsilon\left\|d_{k-1}\right\|\right\}} \\
& -\frac{2\left\|y_{k-1}^{*}\right\|^{2}}{\left(\max \left\{d_{k-1}^{T} y_{k-1}^{*}, \epsilon\left\|d_{k-1}\right\|\right\}\right)^{2}} F_{k}^{T} d_{k-1},
\end{aligned}
$$

where $y_{k-1}^{*}=y_{k-1}+\alpha_{k-1} d_{k-1}$.

Since the definition of parameter $\beta_{k}$ in Algorithm 1(b) could be the same as that in Algorithm 1(a), and the descent property of $d_{k}$ in Algorithm 1(b) is independent of the choices of parameter $\beta_{k}$, we can give several specific versions of Algorithm 1(b) as follows.

Method 4. Consider Algorithm 1(b) with (52).

Method 5. Consider Algorithm 1(b) with

$$
\begin{aligned}
\beta_{k}^{5}= & \frac{F_{k}^{T} y_{k-1}}{\max \left\{\max \left\{d_{k-1}^{T} y_{k-1},-F_{k-1}^{T} d_{k-1}\right\}, \epsilon\left\|d_{k-1}\right\|\right\}} \\
& -\frac{2\left\|y_{k-1}\right\|^{2}}{\left(\max \left\{\max \left\{d_{k-1}^{T} y_{k-1},-F_{k-1}^{T} d_{k-1}\right\}, \epsilon\left\|d_{k-1}\right\|\right\}\right)^{2}} \\
& \times F_{k}^{T} d_{k-1} .
\end{aligned}
$$

Method 6. Consider Algorithm 1(b) with

$$
\beta_{k}^{6}=\frac{F_{k}^{T} y_{k-1}}{\max \left\{d_{k-1}^{T} y_{k-1}, \epsilon\left\|d_{k-1}\right\|\right\}} .
$$

From Lemma 8, we know that a sequence $\left\{F_{k}\right\}$ generated by Algorithm 1 is norm bounded. Then it is easy to verify that the parameters $\beta_{k}$ in Methods 1-6 satisfy Assumption 4. So, from the convergence analysis in Section 3, we know that Methods 1-6 are convergent in the sense that $\liminf _{k \rightarrow \infty}\left\|F_{k}\right\|=0$.

Next, we test the performance of Methods $1-6$ via the following four constrained monotone problems and compare them with the method (abbreviated as CGD_XZ) in [9].

Problem 10 (see [29]). The mapping $F$ is taken as $F(x)=$ $\left(F_{1}(x), \ldots, F_{n}(x)\right)^{T}$, where $F_{i}(x)=e^{x_{i}}-1, i=1, \ldots, n$, and $\Omega=R_{+}^{n}$. 
Problem 11 (see [17]). The mapping $F$ is taken as $F(x)=$ $\left(F_{1}(x), \ldots, F_{n}(x)\right)^{T}$, where

$$
F_{i}(x)=x_{i}-\sin \left(\left|x_{i}-1\right|\right), \quad i=1,2, \ldots, n,
$$

and $\Omega=\left\{x \in R^{n} \mid \sum_{i=1}^{n} x_{i} \leq n, x_{i} \geq 0, i=1,2, \ldots, n\right\}$.

Problem 12 (see [30]). The mapping $F$ is taken as $F(x)=$ $\left(F_{1}(x), \ldots, F_{n}(x)\right)^{T}$, where

$$
\begin{gathered}
F_{1}(x)=x_{1}-e^{\cos \left(\left(x_{1}+x_{2}\right) /(n+1)\right)}, \\
F_{i}(x)=x_{i}-e^{\cos \left(\left(x_{i-1}+x_{i}+x_{i+1}\right) /(n+1)\right)}, \quad i=2,3, \ldots, n-1, \\
F_{n}(x)=x_{n}-e^{\cos \left(\left(x_{n-1}+x_{n}\right) /(n+1)\right)},
\end{gathered}
$$

and $\Omega=R_{+}^{n}$.

Problem 13 (see [31]). The mapping $F$ is given by

$$
F(x)=\left(\begin{array}{cccc}
1 & 0 & 0 & 0 \\
0 & 1 & -1 & 0 \\
0 & 1 & 1 & 0 \\
0 & 0 & 0 & 0
\end{array}\right)\left(\begin{array}{l}
x_{1} \\
x_{2} \\
x_{3} \\
x_{4}
\end{array}\right)+\left(\begin{array}{l}
x_{1}^{3} \\
x_{2}^{3} \\
2 x_{3}^{3} \\
2 x_{4}^{3}
\end{array}\right)+\left(\begin{array}{c}
-10 \\
1 \\
-3 \\
0
\end{array}\right)
$$

and $\Omega=\left\{x \in R^{4} \mid \sum_{i=1}^{4} x_{i} \leq 4, x_{i} \geq 0, i=1,2,3,4\right\}$.

For Methods 1-6 and CGD_XZ method, we set $\sigma=10^{-4}$, $t=0.5$, and $\rho=s_{k}^{T} s_{k} /\left(s_{k}^{T} y_{k}\right)$, where $s_{k}=x_{k+1}-x_{k}$ and $\rho \geq 1 / L$ which is obtained by the monotonicity and the L-Lipschitz continuity of $F$. The stopping criterion is $\left\|F_{k}\right\|_{\infty} \leq 10^{-5}$.

Our computations were carried out using MATLAB R2011b on a desktop computer with an Intel(R) Xeon(R) 2.40 GHZ CPU, 6.00 GB of RAM, and Windows operating system. The numerical results were reported in Tables 1, 2, 3 , and 4 , where the initial points $x_{1}=(10,10, \ldots, 10)^{T}$, $x_{2}=(1,1, \ldots, 1)^{T}, x_{3}=(1,1 / 2, \ldots, 1 / n)^{T}, x_{4}=(0.1,0.1$, $\ldots, 0.1)^{T}, x_{5}=(1 / n, 2 / n, \ldots, 1)^{T}$, and $x_{6}=(1-1 / n, 1-$ $2 / n, \ldots, 0)^{T}$ and Dim, Iter, Nf, and CPU stand for the dimension of the problem, the number of iterations, the number of function evaluations, and the CPU time elapsed in seconds, respectively. Table 5 showed the number that each method solved the test problems with the least iterations, the least function evaluations, and the best time, respectively.

The performance of the seven methods was evaluated using the profiles of Dolan and Morè [32]. That is, we plotted the fraction $P$ of the test problems for which each of the methods was within a factor $\tau$ of the best time. Figures 1-3 showed the performance profiles referring to the number of iterations, the number of function evaluations, and CPU time, respectively. Figure 1 indicated that relative to the number of iterations, Methods 1 and 2 performed best for $\tau$ near 1 . When $\tau \geq 1$, CGD XZ was comparable with Methods 1 and 2 and had a higher number of wins than Methods 3-6. Figure 2 revealed that relative to the number

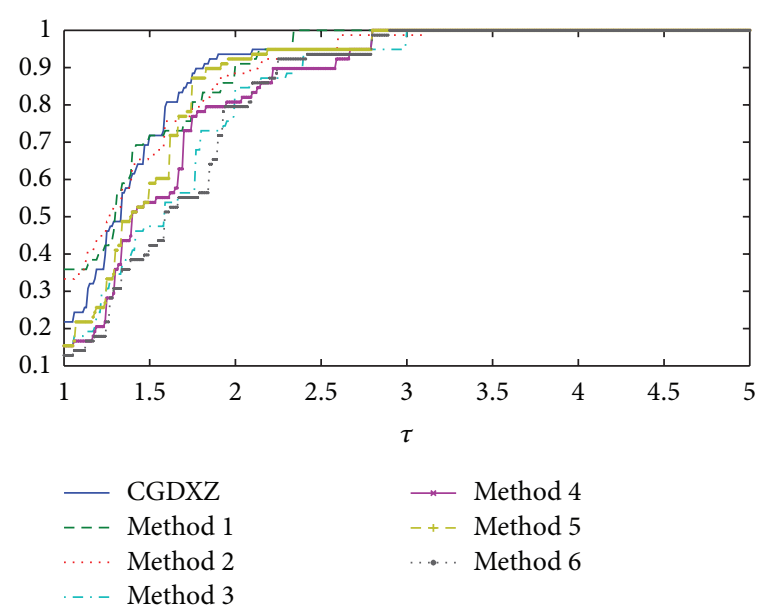

FIGURE 1: Performance profile based on the number of iterations.

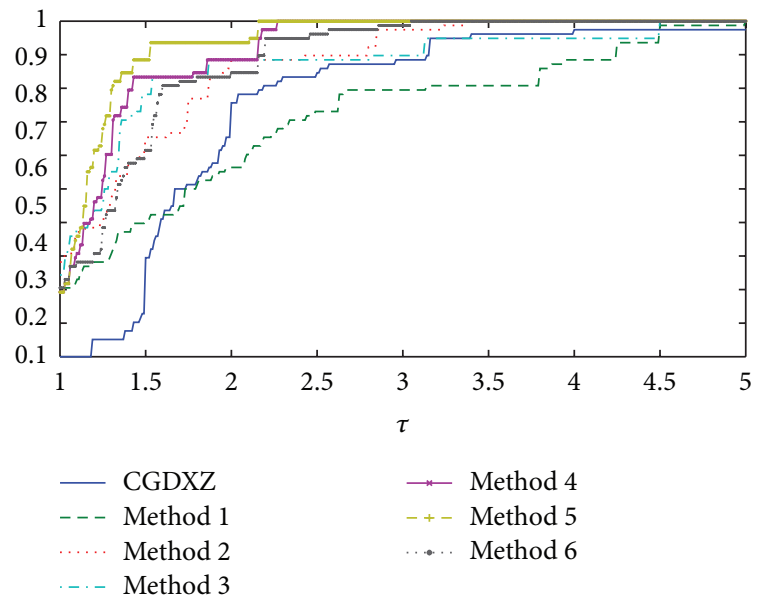

FIGURE 2: Performance profile based on the number of function evaluations.

of function evaluations, Method 2 performed best for $\tau$ near 1. When $\tau \geq 1.6$, Method 5 performed more robust and then Methods 3 and 4 . Figure 3 revealed that relative to the CPU time metric, Method 2 performed best for $\tau$ near 1 . Method 5 performed more robust when $\tau \geq 1$.3, and Methods 2-4 were competitive. While CGD_XZ performed worst, it had a lower number of wins than the rest of the methods. So, from the analysis above, we can conclude that all these methods were efficient to solve these test problems. If we consider the number of wins, Method 2 performed best which is also revealed by Table 5, while from the view of robustness, Method 5 performed best.

\section{Conclusions}

In this paper, we discussed two unified frameworks of some sufficient descent conjugate gradient methods and combined them with the hyperplane projection method of Solodov and Svaiter to solve convex constrained nonlinear monotone equations. The two unified frameworks inherit the advantages 


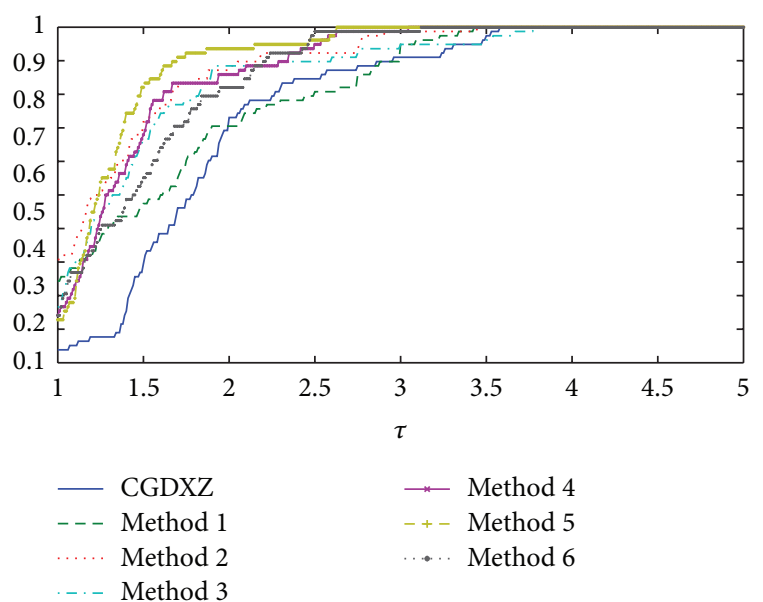

Figure 3: Performance profile based on the CPU time.

of some usual conjugate gradient methods for solving largescale unconstrained minimization problems. That is, they satisfy the sufficient descent condition $F_{k}^{T} d_{k} \leq-c\left\|F_{k}\right\|^{2}$ ( $c>0$ ) independently of any line search, and they do not require $F$ 's Jacobian, then they are suitable to solve large-scale nonsmooth monotone equations. In Section 4, we gave several specific versions of the two unified frameworks and investigated their numerical behaviors over some test problems. From the numerical results, we concluded that these specific versions are efficient.

Let us review problem (1) and introduce a monotone inclusion problem

$$
0 \in T(x)
$$

where the set-value mapping $T: R^{n} \rightarrow 2^{R^{n}}$ is maximal monotone. Obviously, the latter is more general than the former; then, our further investigation is to extend these sufficient descent conjugate gradient methods to solve problem (60).

\section{Conflict of Interests}

The authors declare that there is no conflict of interests regarding the publication of this paper.

\section{Acknowledgment}

This work was supported by the National Science Foundation of China, no. 61373174.

\section{References}

[1] J. M. Ortega and W. C. Rheinboldt, Iterative Solution of Nonlinear Equations in Several Variables, Academic Press, New York, NY, USA, 1970.

[2] M. E. El-Hawary, Optimal Power Flow: Solution Techniques, Requirement and Challenges, IEEE Service Center, Piscataway, NJ, USA, 1996.
[3] A. J. Wood and B. F. Wollenberg, Power Generations, Operations, and Control, John Wiley \& Sons, New York, NY, USA, 1996.

[4] Y.-B. Zhao and D. Li, "Monotonicity of fixed point and normal mappings associated with variational inequality and its application," SIAM Journal on Optimization, vol. 11, no. 4, pp. 962-973, 2001.

[5] M. V. Solodov and B. F. Svaiter, "A globally convergent inexact Newton method for systems of monotone equations," in Reformulation: Nonsmooth, Piecewise Smooth, Semismooth and Smoothing Methods, M. Fukushima and L. Qi, Eds., vol. 22, pp. 355-369, Kluwer Academic Publishers, Dordrecht, The Netherlands, 1999.

[6] G. Zhou and K. C. Toh, "Superlinear convergence of a Newtontype algorithm for monotone equations," Journal of Optimization Theory and Applications, vol. 125, no. 1, pp. 205-221, 2005.

[7] W.-J. Zhou and D.-H. Li, "A globally convergent BFGS method for nonlinear monotone equations without any merit functions," Mathematics of Computation, vol. 77, no. 264, pp. 22312240, 2008.

[8] W. Zhou and D. Li, "Limited memory BFGS method for nonlinear monotone equations," Journal of Computational Mathematics, vol. 25, no. 1, pp. 89-96, 2007.

[9] Y. Xiao and H. Zhu, "A conjugate gradient method to solve convex constrained monotone equations with applications in compressive sensing," Journal of Mathematical Analysis and Applications, vol. 405, no. 1, pp. 310-319, 2013.

[10] W. Cheng, "A PRP type method for systems of monotone equations," Mathematical and Computer Modelling, vol. 50, no. 1-2, pp. 15-20, 2009.

[11] Q.-R. Yan, X.-Z. Peng, and D.-H. Li, "A globally convergent derivative-free method for solving large-scale nonlinear monotone equations," Journal of Computational and Applied Mathematics, vol. 234, no. 3, pp. 649-657, 2010.

[12] Q. Li and D.-H. Li, "A class of derivative-free methods for largescale nonlinear monotone equations," IMA Journal of Numerical Analysis, vol. 31, no. 4, pp. 1625-1635, 2011.

[13] M. Ahookhosh, K. Amini, and S. Bahrami, "Two derivativefree projection approaches for systems of large-scale nonlinear monotone equations," Numerical Algorithms, vol. 64, no. 1, pp. 21-42, 2013.

[14] M. Li, "A LS type method for solving large-scale nonlinear monotone equations," Numerical Functional Analysis and Optimization, 2013.

[15] W. Cheng, Y. Xiao, and Q.-J. Hu, "A family of derivative-free conjugate gradient methods for large-scale nonlinear systems of equations," Journal of Computational and Applied Mathematics, vol. 224, no. 1, pp. 11-19, 2009.

[16] W. La Cruz and M. Raydan, "Nonmonotone spectral methods for large-scale nonlinear systems," Optimization Methods \& Software, vol. 18, no. 5, pp. 583-599, 2003.

[17] L. Zhang and W. Zhou, "Spectral gradient projection method for solving nonlinear monotone equations," Journal of Computational and Applied Mathematics, vol. 196, no. 2, pp. 478-484, 2006.

[18] Z. Yu, J. Lin, J. Sun, Y. Xiao, L. Liu, and Z. Li, "Spectral gradient projection method for monotone nonlinear equations with convex constraints," Applied Numerical Mathematics, vol. 59, no. 10, pp. 2416-2423, 2009.

[19] Y. H. Dai, "Nonlinear conjugate gradient methods," in Wiley Encyclopedia of Operations Research and Management Science, J. J. Cochran, L. A. Cox Jr, P. Keskinocak, J. P. Kharoufeh, and J. C. Smith, Eds., John Wiley \& Sons, 2011. 
[20] W. W. Hager and H. Zhang, "A new conjugate gradient method with guaranteed descent and an efficient line search," SIAM Journal on Optimization, vol. 16, no. 1, pp. 170-192, 2005.

[21] W. W. Hager and H. Zhang, "Algorithm 851: CGDESCENT, a conjugate gradient method with guaranteed descent," ACM Transactions on Mathematical Software, vol. 32, no. 1, pp. 113137, 2006.

[22] G. Yu, L. Guan, and W. Chen, "Spectral conjugate gradient methods with sufficient descent property for large-scale unconstrained optimization," Optimization Methods \& Software, vol. 23, no. 2, pp. 275-293, 2008.

[23] W. Cheng and Q. Liu, "Sufficient descent nonlinear conjugate gradient methods with conjugacy condition," Numerical Algorithms, vol. 53, no. 1, pp. 113-131, 2010.

[24] E. Polak and G. Ribière, "Note sur la convergence de méthodes de directions conjuguées," Revue Francaise Dinformatique et Derecherche Opérationnelle, Série Rouge, vol. 3, no. 16, pp. 3543, 1969.

[25] B. T. Polyak, "The conjugate gradient method in extremal problems," USSR Computational Mathematics and Mathematical Physics, vol. 9, no. 4, pp. 94-112, 1969.

[26] Y.-H. Dai and C.-X. Kou, "A nonlinear conjugate gradient algorithm with an optimal property and an improved Wolfe line search," SIAM Journal on Optimization, vol. 23, no. 1, pp. 296320, 2013.

[27] W. W. Hager and H. Zhang, "The limited memory conjugate gradient method," SIAM Journal on Optimization, vol. 23, no. 4, pp. 2150-2168, 2013.

[28] M. R. Hestenes and E. Stiefel, "Methods of conjugate gradients for solving linear systems," Journal of Research of the National Bureau of Standards, vol. 49, no. 6, pp. 409-436, 1952.

[29] C. Wang, Y. Wang, and C. Xu, "A projection method for a system of nonlinear monotone equations with convex constraints," Mathematical Methods of Operations Research, vol. 66, no. 1, pp. 33-46, 2007.

[30] G. Yu, S. Niu, and J. Ma, "Multivariate spectral gradient projection method for nonlinear monotone equations with convex constraints," Journal of Industrial and Management Optimization, vol. 9, no. 1, pp. 117-129, 2013.

[31] N. Yamashita and M. Fukushima, "Modified Newton methods for solving a semismooth reformulation of monotone complementarity problems," Mathematical Programming, vol. 76, no. 3, pp. 469-491, 1997.

[32] E. D. Dolan and J. J. Moré, "Benchmarking optimization software with performance profiles," Mathematical Programming, vol. 91, no. 2, pp. 201-213, 2002. 


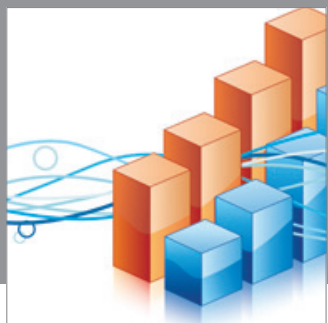

Advances in

Operations Research

mansans

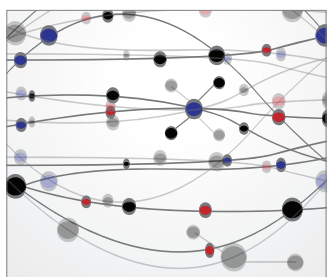

The Scientific World Journal
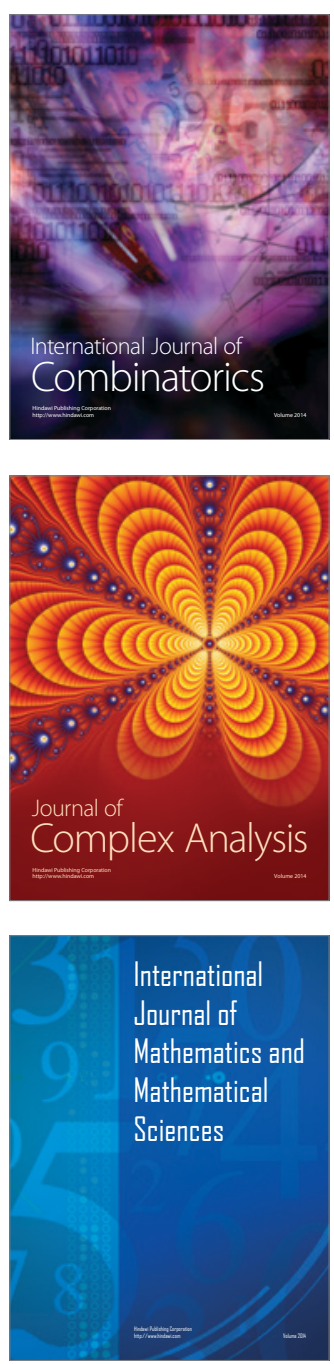
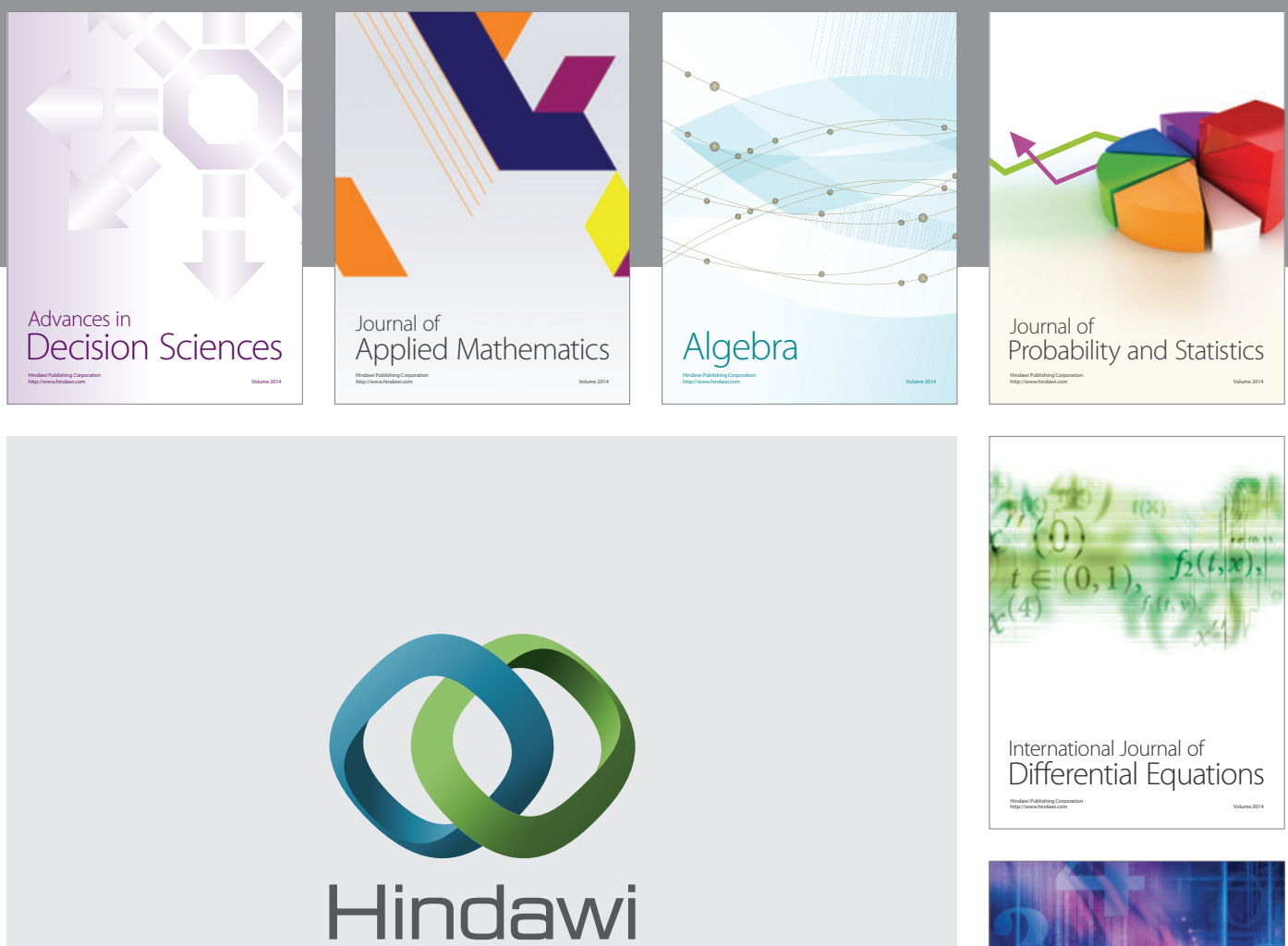

Submit your manuscripts at http://www.hindawi.com
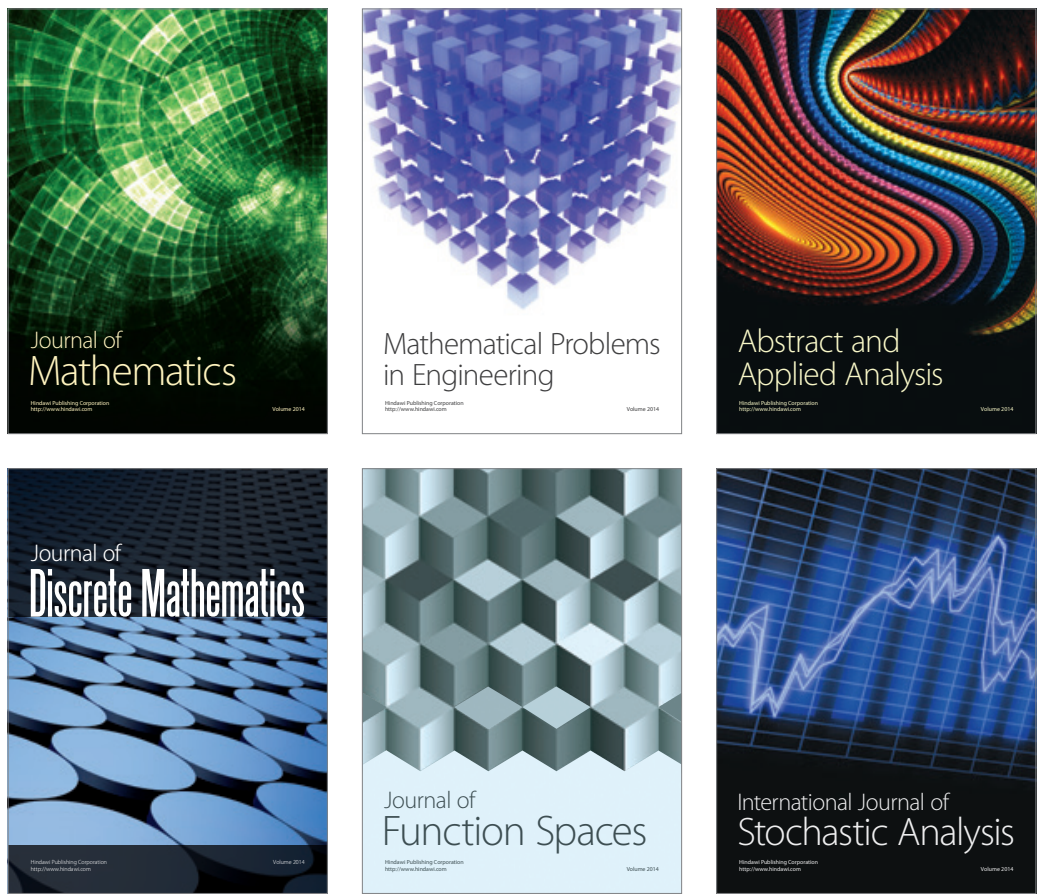

Journal of

Function Spaces

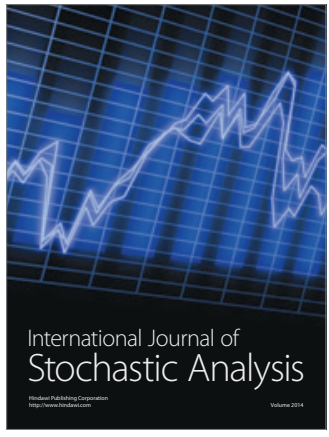

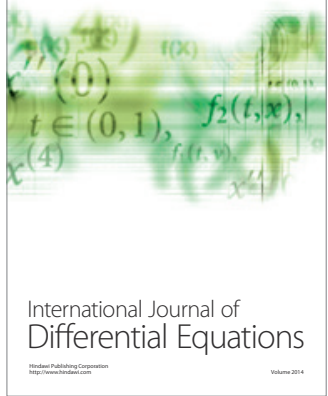
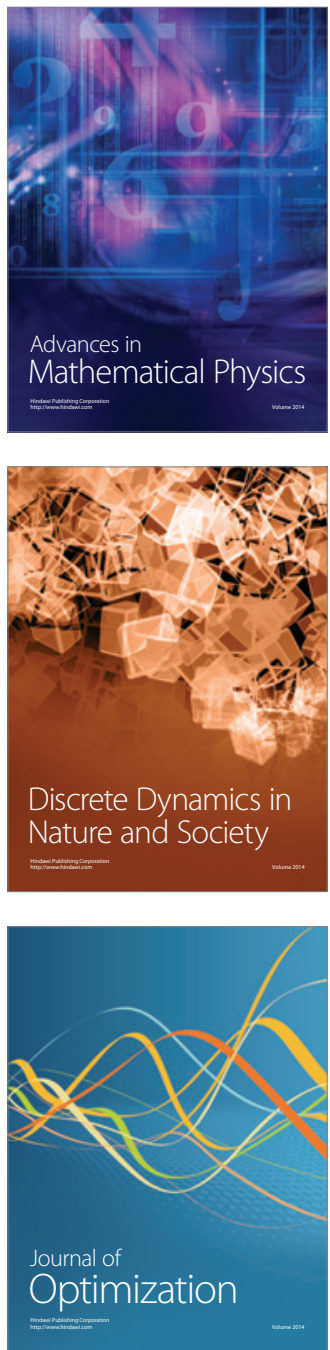